\title{
La antiguiedad de los indefinidos despedidos en España. ¿Hay un antes y un después de la reforma laboral de 2010?
}

\author{
Begoña Cueto ${ }^{\mathrm{a}, *}$ y Vanesa Rodríguez ${ }^{\mathrm{b}}$ \\ a Departamento de Economía Aplicada, Universidad de Oviedo, Oviedo, España \\ b Departamento de Sociología, Universidad de Oviedo, Oviedo, España
}

Recibido el 28 de noviembre de 2012; aceptado el 27 de mayo de 2013

Disponible en Internet el 20 de septiembre de 2013

\section{CÓDIGOS JEL \\ K31, J63, J53 \\ PALABRAS CLAVE \\ Despido; \\ Antigüedad; \\ Reforma laboral}

\section{JEL CLASSIFICATION \\ $\mathrm{K} 31, \mathrm{~J} 63$, J53}

\section{KEYWORDS}

Dismissal;

Seniority;

Labour market reform

\begin{abstract}
Resumen Las reformas laborales de los últimos años han afectado al sistema de despido, entre otros aspectos, clarificando las causas del mismo y tratando de reducir su coste efectivo. Entre sus efectos, cabe señalar la posibilidad de reducir los incentivos para despedir a los trabajadores con menor antigüedad, cambiando, por tanto, la aplicación de la regla LIFO (last in, first out). A partir de los datos de la Muestra Continua de Vidas Laborales se han analizado las características de los trabajadores despedidos en el periodo 2005-2011, encontrando que la mayor parte de los contratos que se terminan de forma no voluntaria tienen una duración inferior a los 2 años. Sin embargo, en los años 2010 y 2011 se produce un cambio, aumentando los despidos en contratos de duración superior a 2 años.

(C) 2012 Asociación Cuadernos de Economía. Publicado por Elsevier España, S.L. Todos los derechos reservados.
\end{abstract}

\begin{abstract}
Job tenure of dismissed workers in Spain. Is there a 'before and after' the 2010 labour market reform?
\end{abstract}

\begin{abstract}
The latest labour market reforms in Spain have affected legal dismissal regulations by clarifying causes, and reducing costs, among other aspects. Among the expected effects of these legal changes is the weakening the 'last in, first out' rule in dismissals of workers with open-ended contracts. Using data from the Longitudinal Working Lives Sample from 2005 to 2011, the empirical analysis shows that the majority of contracts that are terminated nonvoluntarily were in the job for less than two years. However, this pattern changes in 2010 and 2011 , with the proportion of dismissals increasing in permanent workers who have been in the same job for more than two years.
\end{abstract}

(c) 2012 Asociación Cuadernos de Economía. Published by Elsevier España, S.L. All rights reserved.

\section{Introducción}

* Autor para correspondencia.

Correo electrónico: bcueto@uniovi.es (B. Cueto).
Durante los 3 últimos años se han realizado numerosos cambios en la regulación del mercado de trabajo en España. 
Las reformas de los años 2010, 2011 y 2012 han afectado a varios aspectos, entre ellos el sistema de despido. La diferencia en las indemnizaciones entre contratos indefinidos y temporales, el uso estratégico de la Ley 45/2002 y la correcta definición de las causas objetivas para el despido económico, entre otros temas, han sido objeto de numerosos debates y estudios.

La necesidad de las reformas se planteó, entre otras razones, por un uso desvirtuado del sistema de despido (Malo, 2005; García Martínez y Malo, 2007). El conocido como «despido exprés» suponía un uso excesivo del despido improcedente como forma de evitar procesos largos e incertidumbre a cambio de abonar una indemnización mayor que la que correspondería al uso de un despido procedente o por causa económica. Así, si una empresa tenía que prescindir de trabajadores, a la hora de decidir a cuáles despedir el criterio de antigüedad se convierte en prioritario, pues un menor tiempo trabajado en la empresa supone una menor indemnización. Por tanto, se tendía a aplicar la regla LIFO, «last in, first out» (el último en entrar es el primero que sale), no solo para los trabajadores temporales, sino también para los indefinidos (Malo, 2010a; Malo y González Sánchez, 2010). Esta regulación favorecía a los trabajadores permanentes con una antigüedad alta, generando una elevada desigualdad ante el despido y en la protección de desempleo, cuya duración también depende del tiempo cotizado (Desdentado, 2010). Los despidos se decidían, pues, con un criterio casi exclusivamente monetario, sin que la productividad de los trabajadores y su capacidad para ayudar a la empresa en momentos de crisis sean razones esenciales a tener en cuenta para tomar decisiones sobre qué trabajadores mantener y cuáles no.

El objetivo con el que se plantea este artículo es analizar las características de los trabajadores despedidos en el periodo 2005-2011 a partir de los datos de la Muestra Continua de Vidas Laborales (MCVL). En este periodo, analizaremos la antigüedad de este grupo de trabajadores y veremos algunos efectos derivados tanto de la crisis que empieza en 2007, como de la reforma de 2010. No obstante, hay que tener en cuenta que no se trata de una evaluación de esta reforma, dado que no se lleva a cabo un análisis de causalidad, sino que se presenta la evolución de ciertos indicadores que están relacionados con los cambios habidos en los años 2008 a 2011. Previamente se presenta la información relativa a los flujos de salida del mercado de trabajo. Estos datos nos permitirán dar cuenta de la magnitud de los despidos en España, incluso en los años previos a la crisis económica, y de los efectos de esta sobre la destrucción de empleo.

\section{Flujos de salida del mercado de trabajo}

Los datos sobre flujos de entrada y salida del mercado de trabajo en España son incompletos, imposibilitando un análisis detallado de estas variables para el mercado laboral español o de los efectos que sobre ellas puedan tener los cambios normativos. Mientras que para conocer el número de ocupados en un determinado momento podemos acudir a varias fuentes (Encuesta de Población Activa; Afiliaciones a la Seguridad Social; Encuesta de Coyuntura Laboral), en el caso de los flujos, únicamente la Encuesta de Coyuntura

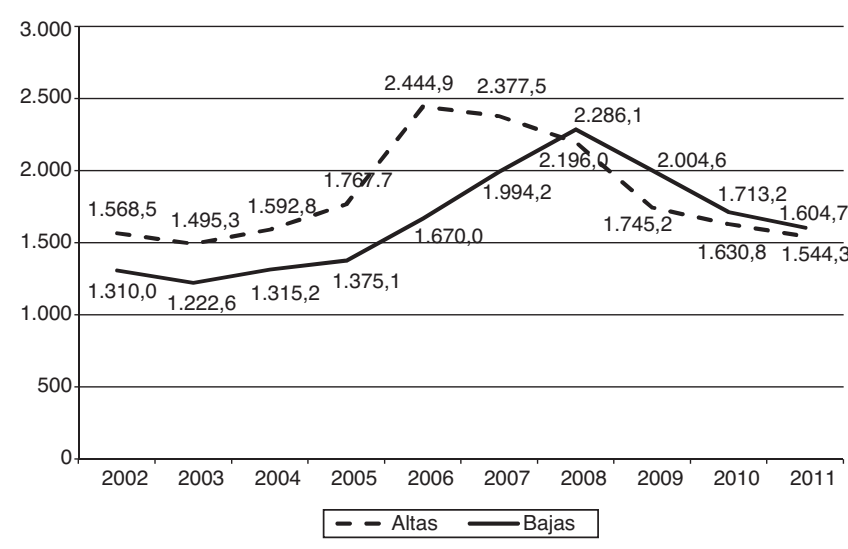

Figura 1 Altas y bajas de efectivos laborales contratados de forma indefinida (se incluyen las modificaciones de contrato, en miles).

Fuente: Encuesta de Coyuntura Laboral (Ministerio de Empleo y Seguridad Social).

Laboral (ECL) nos aporta información en este sentido ${ }^{1}$. En la figura 1 se muestran los datos correspondientes al periodo 2005-2011. Se puede observar que hasta el año 2007 el saldo neto era positivo, lo que suponía creación de empleo indefinido. En cambio, desde dicho año el número de bajas supera el de altas, comenzado un periodo de intensa destrucción de puestos de trabajo que alcanza su máximo en 2009, con un saldo neto de casi 260.000 trabajadores. Aunque suele destacarse el efecto de la crisis económica sobre las bajas, resulta también muy importante su efecto sobre las altas, que, como se aprecia en la figura, ya empieza en 2007 , al reducirse un 2,8\% respecto al año anterior, y es especialmente intenso en 2009 , con una caída en el número de altas de más del $20 \%$ respecto al año anterior. Así, aunque a partir de este año también se reduce el número de bajas, el saldo neto continúa siendo negativo.

La desventaja de los datos de la ECL para atender el objetivo propuesto en este artículo es que no se publica la causa de la baja ni el tipo de contratación más allá de la diferencia entre temporales e indefinidos ${ }^{2}$. Para tener información sobre despidos, debemos acudir a las estadísticas de Expedientes de Regulación de Empleo o a las Altas en el Sistema de Prestaciones por desempleo. La primera incluye únicamente los despidos colectivos (extinción, suspensión o reducción), mientras que la segunda también aporta datos sobre despidos individuales.

En la figura 2 se muestra el número de trabajadores afectados por ERE. Esta fórmula de despido se ha venido utilizando de forma relativamente escasa en comparación con los despidos individuales debido a distintas disfuncionalidades (Malo, 2005; García-Serrano et al., 2010a; Malo y González Sánchez, 2010). Por ejemplo, la necesaria autorización administrativa previa, eliminada por el Real Decreto

\footnotetext{
${ }^{1} \mathrm{El}$ ámbito sectorial de la ECL incluye industria, construcción y servicios (excluyendo Administración Pública, Defensa, Seguridad Social, Organismos Extraterritoriales y Organizaciones religiosas).

2 Hospido y Jimeno (2011) ofrecen datos sobre la causa de baja laboral con datos de la ECL. No obstante, es una explotación específica de los microdatos de dicha encuesta.
} 


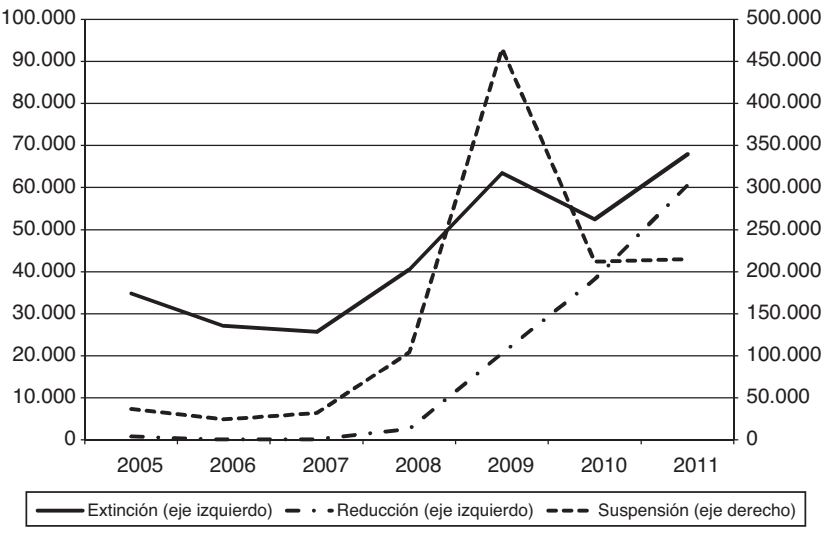

Figura 2 Trabajadores afectados por expedientes autorizados/comunicados.

Fuente: Anuario de Estadísticas Laborales (Ministerio de Empleo y Seguridad Social).

Ley $3 / 2012$, que introducía incertidumbre en el empresario a la hora de poner en marcha este procedimiento, puesto que la posterior autorización de la autoridad laboral dependía en gran parte del acuerdo previo con los trabajadores (lo que podía hacer que la consulta con los trabajadores se convirtiera en un proceso de negociación que elevara la indemnización a pagar en un momento en el que la empresa se encontraba en una mala situación económica). Esta situación hacía que fuera más factible un ERE en momentos de bonanza que de crisis, puesto que en los primeros la empresa podía pagar una mayor indemnización a cambio del acuerdo, mientras que en momentos de crisis retrasaba lo más posible el ERE debido a su probable elevado coste, lo que hacía que cuando lo aplicase ya no solucionase la mala situación de la empresa, sino que más bien fuese el primer paso del cierre de esta (Malo, 2012a) A pesar de estas dificultades para llevarlos a cabo, se puede apreciar el fuerte aumento experimentado a partir de 2008.

Las cifras correspondientes a altas en el sistema de prestaciones por desempleo se muestran en la tabla 1, diferenciando entre la causa del despido y si el trabajador tiene derecho a prestación de nivel contributivo o asistencial ${ }^{3}$. Se puede apreciar que el número de despidos es elevado, incluso en años de expansión económica ${ }^{4}$. La magnitud de las cifras sugiere que el despido individual se utiliza como un instrumento de ajuste de la plantilla de las empresas, tanto en crisis como en periodos de expansión (García-Serrano, 2011). En los años 2003 y 2004 parece que se extiende el uso de los despidos que tienen su causa en el artículo 1 de la Ley $45 / 2002$, con un crecimiento del 147,5 y del $58,5 \%$ respecto al año anterior, en cada año. En 2005 se produce

\footnotetext{
${ }^{3}$ En este sentido, hay que tener en cuenta que no se incluyen los trabajadores despedidos que no tienen acceso al nivel asistencial de la prestación por desempleo por no haber cotizado el tiempo suficiente. Por tanto, el número de despidos se infravalora.

4 Toharia y Malo (2009) señalan que «la reforma de 2002 elevó el número de despidos, que pasó del $4 \%$ de los asalariados indefinidos privados en 2000 al 5,5\% en todos los años comprendidos entre 2002 y 2007 , para aumentar posteriormente, ya con la crisis, al $7 \%$ en el año 2008».
}

un descenso del 10,4\%, para volver a incrementarse entre 2006 y 2009.

Los efectos de la crisis se ven con claridad, con un aumento de casi el $20 \%$ en el número de despidos totales en 2008 , que asciende al $56,3 \%$ en el caso de los despidos individuales. El máximo se alcanza al año siguiente, superando el millón de despidos individuales y casi duplicando, respecto al año anterior, la cifra de despidos colectivos. Aunque en 2010 y 2011 se ha reducido el número total, se mantiene en cifras superiores a los 800.000 anuales.

La gran mayoría de las altas en el sistema de prestaciones por desempleo tienen su origen en el nivel contributivo; es decir, se trata de personas que han trabajado al menos durante un año en su último episodio de empleo, o que habiendo trabajado menos tiempo no habían agotado su anterior prestación contributiva o que han trabajado al menos un año desde su última prestación por desempleo (aunque haya sido mediante diversos contratos con episodios de desempleo intercalados) ${ }^{5}$. En el caso de los despidos individuales, este grupo supone más del $95 \%$ en todos los casos, excepto en 2009. Hay que tener en cuenta que en este año, por una parte, se alcanza el máximo número de despidos $y$, por otra, en el número de altas con origen en el nivel asistencial se incluyen las producidas en el Programa Temporal de Prestación por Desempleo e Inserción (PRODI), lo que sin duda afecta al porcentaje que representa cada tipo de alta sobre el total. Para los despidos colectivos, el número de altas con origen en el nivel contributivo ha tendido a aumentar, especialmente en los 2 últimos años, en los que casi el $100 \%$ se corresponden con este grupo.

Por último, en la figura 3 se presenta la distribución de los despidos individuales según causa del mismo. La forma más utilizada con diferencia es el artículo 1 de la Ley 45/2002. Como ya han señalado Malo y Toharia (2008), en torno a las tres cuartas partes de todos los despidos se resuelven por esta vía. La aplicación de esta Ley ha dado lugar a la denominación «despido exprés» para explicar cómo el proceso de despido se convierte en rápido (como máximo $48 \mathrm{~h}$ ) y fácil (carta de despido, reconocimiento de improcedencia y depósito de la indemnización) a cambio de una indemnización más elevada (45 días de salario por año trabajado) que si se opta por otra forma de despido. Esta distorsión en el sistema de despido también ha ocasionado que los despidos por causas objetivas representen un pequeño porcentaje del total, incluso en periodos de crisis económica, donde estaría justificado el uso de esta forma de despido.

La reforma laboral de $2010^{6}$ supuso una nueva redacción de la causa económica para el despido, tratando de conseguir que la indemnización por despido económico procedente fuera la nueva referencia del sistema y, así, corregir la distorsión mencionada previamente (GarcíaSerrano et al., 2010b). El objetivo era que la nueva definición de causa económica permitiese, efectivamente, que cuando hubiera causa económica se pudieran hacer

\footnotetext{
${ }^{5}$ El nivel asistencial es tanto para individuos que han agotado la prestación contributiva como para los que no han cubierto el periodo mínimo para acceder a esta, siempre y cuando cumplan determinados requisitos (pueden consultarse en: www.sepe.es/contenido/prestaciones/pdf/f_sub_desemp.pdf).

${ }^{6}$ Real Decreto-Ley $10 / 2010$ y Ley 35/2010.
} 
Tabla 1 Altas en el sistema de prestaciones por desempleo por despido (altas iniciales y reanudaciones)*

\begin{tabular}{|c|c|c|c|c|c|c|c|c|c|c|}
\hline & 2002 & 2003 & 2004 & 2005 & 2006 & 2007 & 2008 & 2009 & 2010 & 2011 \\
\hline Despido (art. $1 .^{\circ}$ Ley $45 / 2002$ ) & 100.906 & 249.727 & 395.901 & 346.222 & 408.099 & 484.221 & 745.736 & 869.252 & 611.875 & 545.849 \\
\hline Causas objetivas (art. 52 a, b, c, d ET) & 68.292 & 47.800 & 51.588 & 51.473 & 48.229 & 46.938 & 96.711 & 167.220 & 154.981 & 210.078 \\
\hline Acta de conciliación & 143.918 & 47.800 & 35.196 & 32.311 & 24.544 & 16.071 & 14.695 & 13.996 & 5.796 & 4.016 \\
\hline Sentencia Juzgado de lo Social & 18.907 & 9.735 & 9.253 & 10.939 & 10.109 & 8.633 & 11.770 & 21.848 & 17.660 & 13.175 \\
\hline Total despidos individuales & 332.023 & 355.062 & 491.938 & 440.945 & 490.981 & 555.863 & 868.912 & 1.072 .316 & 790.312 & 773.118 \\
\hline Por despidos colectivos & 40.012 & 40.297 & 32.648 & 38.602 & 37.448 & 38.957 & 46.628 & 86.610 & 72.040 & 80.667 \\
\hline \multicolumn{11}{|c|}{ Nivel contributivo } \\
\hline & 2002 & 2003 & 2004 & 2005 & 2006 & 2007 & 2008 & 2009 & 2010 & 2011 \\
\hline Despido (art. $1 .^{\circ}$ Ley 45/2002) & 98.179 & 243.572 & 298.666 & 332.122 & 387.915 & 459.904 & 708.678 & 763.618 & 586.474 & 519.696 \\
\hline Causas objetivas (art. $52 \mathrm{a}, \mathrm{b}, \mathrm{c}, \mathrm{d}$ ET) & 66.272 & 56.715 & 50.835 & 48.952 & 44.926 & 43.519 & 92.497 & 154.908 & 152.812 & 207.247 \\
\hline Acta de conciliación & 135.121 & 46.091 & 34.558 & 28.751 & 19.881 & 11.303 & 10.257 & 8.686 & 5.666 & 3.890 \\
\hline Sentencia Juzgado de lo Social & 16.802 & 9.276 & 9.010 & 10.024 & 8.927 & 7.402 & 10.520 & 19.363 & 17.365 & 12.865 \\
\hline Total despidos individuales & 316.374 & 355.654 & 393.069 & 419.849 & 461.649 & 522.128 & 821.952 & 946.575 & 762.317 & 743.698 \\
\hline Por despidos colectivos & 37.841 & 39.887 & 32.447 & 31.702 & 28.073 & 141.307 & 38.561 & 77.060 & 71.897 & 80.531 \\
\hline \multicolumn{11}{|c|}{ Nivel asistencial } \\
\hline & 2002 & 2003 & 2004 & 2005 & 2006 & 2007 & 2008 & $2009^{*}$ & 2010 & 2011 \\
\hline Despido (art. $1 .^{\circ}$ Ley 45/2002) & 2.711 & 6.130 & 7.235 & 14.100 & 20.184 & 24.317 & 37.058 & 105.634 & 25.401 & 26.153 \\
\hline Causas objetivas (art. $52 \mathrm{a}, \mathrm{b}, \mathrm{c}, \mathrm{d}$ ET) & 1.255 & 869 & 753 & 2.521 & 3.303 & 3.419 & 4.214 & 12.312 & 2.169 & 2.831 \\
\hline Acta de conciliación & 2.558 & 883 & 638 & 3.560 & 4.663 & 4.768 & 4.438 & 5.310 & 130 & 126 \\
\hline Sentencia Juzgado de lo Social & 471 & 196 & 243 & 915 & 1.182 & 1.234 & 1.250 & 2.485 & 295 & 310 \\
\hline Total despidos individuales & 6.995 & 8.078 & 8.869 & 21.096 & 29.332 & 33.738 & 46.960 & 125.741 & 27.995 & 29.420 \\
\hline Por despidos colectivos & 222 & 243 & 201 & 6.900 & 9.375 & 9.949 & 8.067 & 9.550 & 143 & 136 \\
\hline
\end{tabular}

Fuente: Anuario de Estadísticas Laborales (Ministerio de Empleo y Seguridad Social).

"No se han incluido las categorías «despido en periodo de prueba» ni otros despidos. 


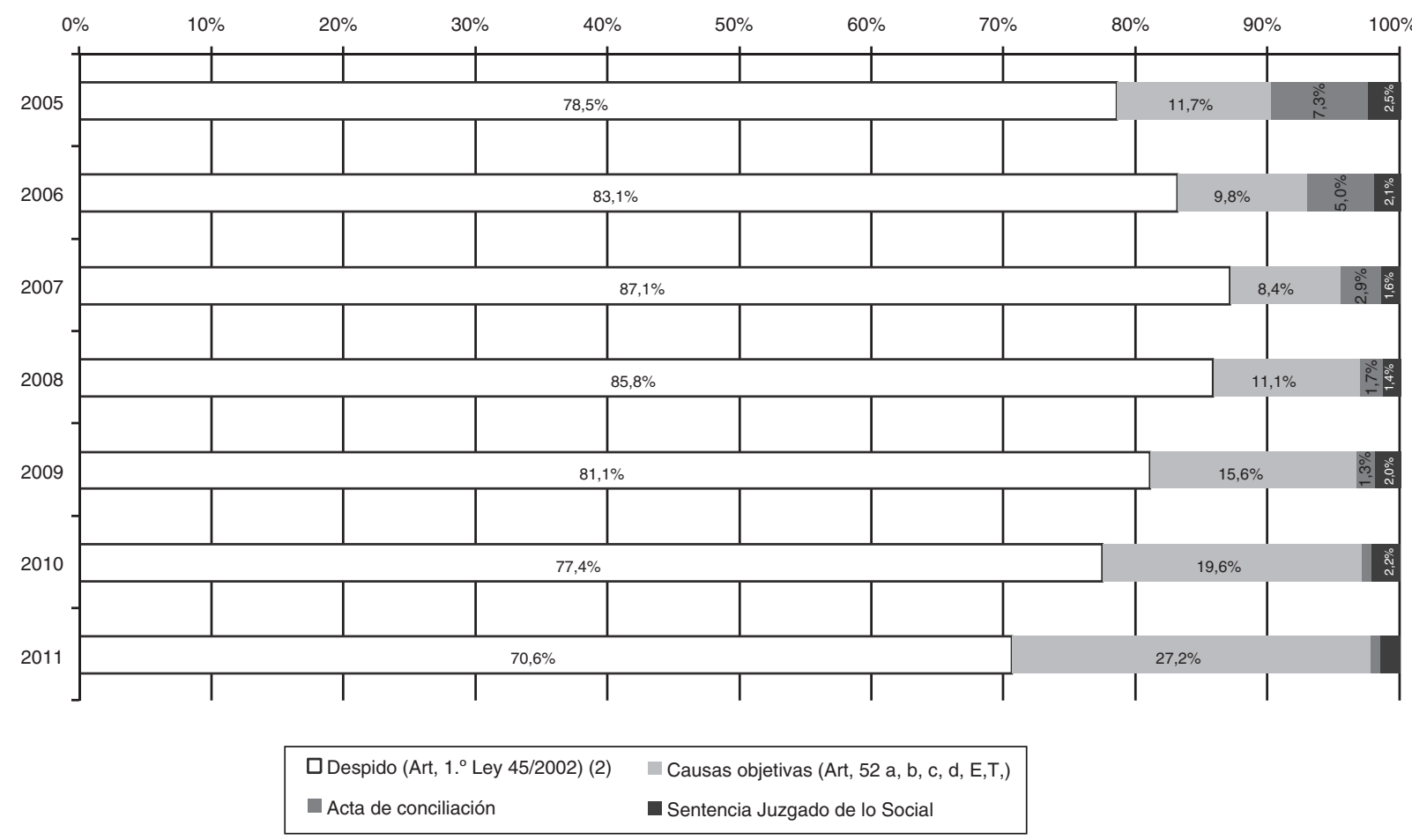

Figura 3 Altas en el sistema de prestaciones por desempleo por despido individual (altas iniciales y reanudaciones). Fuente: Anuario de Estadísticas Laborales (Ministerio de Empleo y Seguridad Social).

despidos económicos de forma sencilla. La reforma llevada a cabo en $2012^{7}$ sigue en la misma dirección, redefiniendo de nuevo las causas del despido económico y eliminando el «despido exprés» (para más información, consultar Malo, 2012a).

En todos los años considerados, los despidos por la vía de la Ley 45/2002 superan las tres cuartas partes del total, aumentando ligeramente hasta 2007 y reduciéndose a partir de dicho año, y en especial en 2011, año en el que suponen el 70,6\% del total. Este cambio ha venido acompañado de una reducción de la proporción de despidos por acta de conciliación administrativa (que pasan del 7,3\% en 2005 al 0,5\% en 2011) y de un fuerte incremento del peso de los despidos por causas económicas.

Aunque el tiempo trascurrido desde la aplicación de la reforma de 2010 es aún corto para valorar su alcance, sí parece que la causa económica se utiliza en mayor medida a partir del año 2010, aunque no podemos señalar que se deba al cambio normativo. No obstante, el hecho de que la reforma de 2010 no haya sido en el margen, es decir, que afecte a todos los trabajadores, no solo a los nuevos entrantes, favorece los efectos inmediatos de la misma (Malo, 2010b).

\section{Las bajas de contratos indefinidos en la Muestra Continua de Vidas Laborales}

El objetivo con el que se ha planteado este artículo es analizar la antigüedad media de los trabajadores despedidos,

\footnotetext{
7 Real Decreto-Ley 3/2012 y Ley 3/2012.
}

con el objetivo de identificar si la crisis y la reforma laboral de 2010 afectan al uso de la regla LIFO en los despidos ${ }^{8}$. Para ello se utilizarán los datos de la Muestra Continua de Vidas Laborales (MCVL). Para las ediciones 2005 a 2011 de la MCVL se han seleccionado las bajas de contratos indefinidos en cada uno de dichos años, indicando si se trata de bajas involuntarias o por expediente de regulación de empleo.

Para responder al objetivo planteado en el artículo nos centramos en los contratos indefinidos a tiempo completo, excluyendo del análisis los contratos a tiempo parcial y también los contratos indefinidos para personas con discapacidad. Respecto a los primeros, son contratos atípicos, con características diferentes a la contratación a tiempo completo $y$, en cuanto a los segundos, tienen una regulación diferente por tratarse de un colectivo con problemas de integración laboral. En resumen, estudiaremos 4 tipos de contratos indefinidos: ordinarios, conversiones, iniciales de fomento del empleo estable y conversiones de fomento del empleo estable? .

La MCVL nos aporta información sobre la causa de baja del contrato, lo que nos permitirá distinguir los despidos del resto de motivos de cese de la actividad laboral. Así, diferenciaremos entre bajas no voluntarias, bajas voluntarias

\footnotetext{
${ }^{8}$ Con los datos disponibles en este momento no es posible analizar los efectos de la reforma de 2012, puesto que la última oleada de la MCVL corresponde a 2011.

${ }^{9}$ La diferenciación por tipos de contrato resulta interesante dado que se ha puesto de manifiesto que la duración de los contratos indefinidos que se acogen a las medidas de fomento de la contratación indefinida es menor, en especial si se trata de contratos iniciales bonificados (Cebrián et al., 2011a, b).
} 
Tabla 2 Contratos indefinidos en vigor (incluye los vigentes y los terminados) y bajas en cada año (datos elevados)

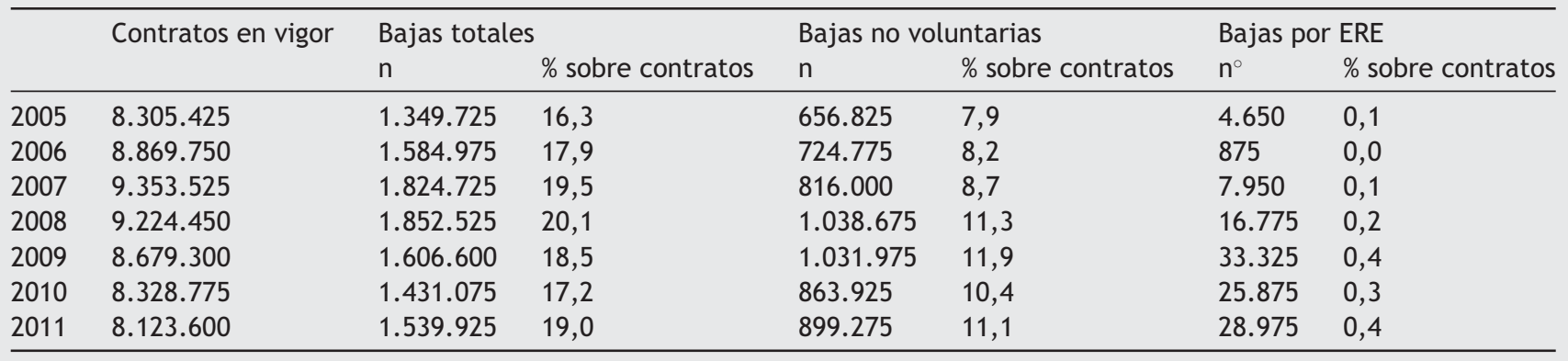

Fuente: Muestra Continua de Vidas Laborales (ediciones 2005 a 2011).

y bajas por ERE en caso de extinción (las cuales no están incluidas en la primera categoría). Tal y como se ha realizado la selección, la muestra a partir de la cual se llevará a cabo el análisis planteado presenta las características de la tabla $2^{10}$. Como cabía esperar, el número más alto de contratos indefinidos vigentes se registra en el año 2007, mientras que las bajas alcanzan su máximo en 2008. No obstante, las bajas no voluntarias registran su valor más elevado (en términos porcentuales) en 2009.

$\mathrm{Si}$ atendemos al porcentaje que suponen las bajas sobre el total de contratos indefinidos vigentes en cada momento, se observa que aumentan hasta 2008, disminuyendo en 2009 y 2010 . Este descenso se debe fundamentalmente a la reducción de las bajas voluntarias, mientras que aumenta la proporción de bajas no voluntarias (despidos) y por pase a regulación de empleo. Así, en el caso de los despidos, el máximo (en términos porcentuales) se alcanza en 2009 (como ya se veía en los datos de altas en prestaciones en la sección anterior), y aunque los trabajadores afectados por ERE son una pequeña proporción, se multiplican por 4 en los años considerados.

Como se puede observar en la figura 4, la principal causa de fin de un contrato indefinido es la baja no voluntaria, es decir, el despido que, además, ha aumentado su peso en el periodo considerado, suponiendo menos de la mitad entre 2005 y 2007 y alcanzando un máximo del 64,4\% del total de bajas en 2009. Las bajas voluntarias se han reducido notablemente, desde casi el $40 \%$ en los años de crecimiento a menos del $15 \%$ en los 3 últimos años considerados. El resto de causas han aumentado su peso, pasando del 15,1 al 26,4\%. Entre ellas se encuentran las bajas por pase a situación de pensionista, por excedencia por el cuidado de hijos, por agotamiento de la incapacidad temporal, u otra. Se puede destacar el aumento de las bajas por pase a regulación de empleo o despido colectivo, que, suponiendo un porcentaje casi nulo en los primeros años considerados, llegan al $2 \%$ en 2011.

\footnotetext{
10 En este cuadro y en las figuras que presentan cifras en términos absolutos, los datos de la MCVL se han elevado. El factor de elevación es 25 , tal y como se indica en las instrucciones de uso de los datos (véase http://www.segsocial.es/Internet_1/Estadistica/Est/Muestra_Continua_de_Vidas_ Laborales/Descripci_n_de_ficheros_y_variables/MCVLSINDATOS/ index.htm).
}

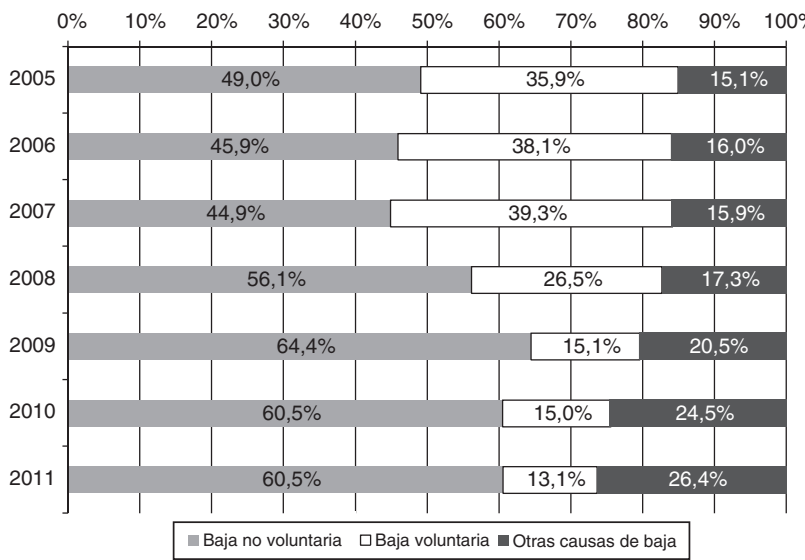

Figura 4 Distribución de las bajas de contratos indefinidos según año de la baja y razón de la misma.

Fuente: Muestra Continua de Vidas Laborales (ediciones 2005 a 2011).

\subsection{Bajas según tipo de contrato indefinido}

En la figura 5 se muestra el número de bajas en cada año considerado en función del tipo de contrato. En primer lugar, se puede señalar que el número máximo de bajas se produce en los años 2007 y 2008, debido al crecimiento del número de bajas de contratos indefinidos ordinarios y de conversión. Las bajas de contratos acogidos al programa de fomento del empleo estable (FEE) -ya sea iniciales o conversiones- se reducen en el periodo considerado, especialmente en el caso de las conversiones que, en los 2 últimos años, no llegan a 1.000 .

En la parte derecha de la figura se ha representado el porcentaje que suponen las bajas no voluntarias sobre el total de cada tipo de contrato. En los 3 primeros años (2005 a 2007) hay una tendencia decreciente en los 4 tipos considerados. Por otra parte, existe una ordenación de forma que el mayor porcentaje de bajas no voluntarias se da en los contratos iniciales, especialmente los de fomento del empleo estable, y la tasa más baja se encuentra en las conversiones de dicho programa. A partir de 2008 se produce un incremento sustancial de las bajas no voluntarias, que pasan, para el total de contratos, del $45 \%$ en 2007 al $64 \%$ en 2009. En estos 2 años son los contratos indefinidos ordinarios los que presentan la menor tasa de salida involuntaria, con una diferencia de 10 puntos porcentuales respecto a las 

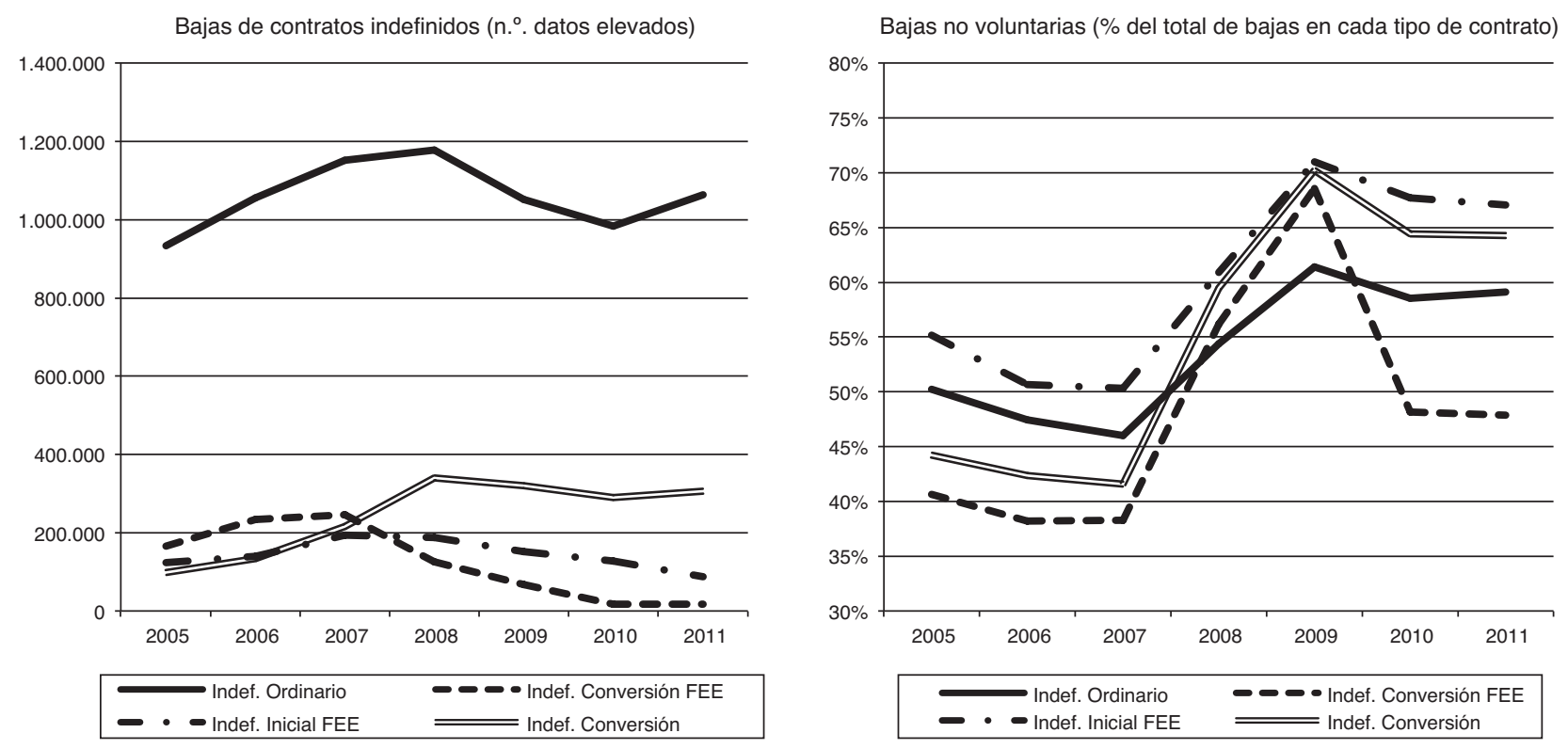

Figura 5 Bajas de contratos indefinidos según año de la baja y tipo de contrato.

Fuente: Muestra Continua de Vidas Laborales (ediciones 2005 a 2011).

otras 3 categorías en el año 2009. Esta brecha se mantiene en los años siguientes, aunque en niveles ligeramente más bajos. No obstante, hay que señalar la caída en la proporción de bajas involuntarias en los contratos indefinidos conversiones de FEE que en 2010 y 2011 no llega al 50\%, si bien hay que tener en cuenta el pequeño tamaño de este grupo en dichos años, como se ha señalado en el párrafo anterior.

\section{La antiguiedad de los trabajadores despedidos en el periodo 2005-2011}

En este apartado se analiza la duración de los contratos indefinidos finalizados de forma involuntaria en el periodo 2005-2011. Nuestra población de referencia, por tanto, incluye todos los ceses involuntarios de contratos indefinidos. En la figura 6 se puede observar que, entre 2005 y 2008 , la antigüedad media presenta una ligera tendencia decreciente, de forma que pasa de 3,7 años a 3,3. A partir de 2009, esta duración media de los contratos indefinidos terminados de forma involuntaria comienza una tendencia ascendente, de forma que en 2011 se alcanzan los 5 años. En la parte derecha de la figura 6 se ha representado el porcentaje de contratos indefinidos finalizados con una duración inferior a 2 años y el correspondiente a los de duración superior a los 10 años. Se puede apreciar que los primeros llegaron a suponer más de la mitad del total de bajas no voluntarias en los años 2007 y 2008, para caer de forma drástica a partir de dicho bienio, suponiendo el 37,4\% del total en 2011. En cambio, aumenta la proporción de contratos terminados
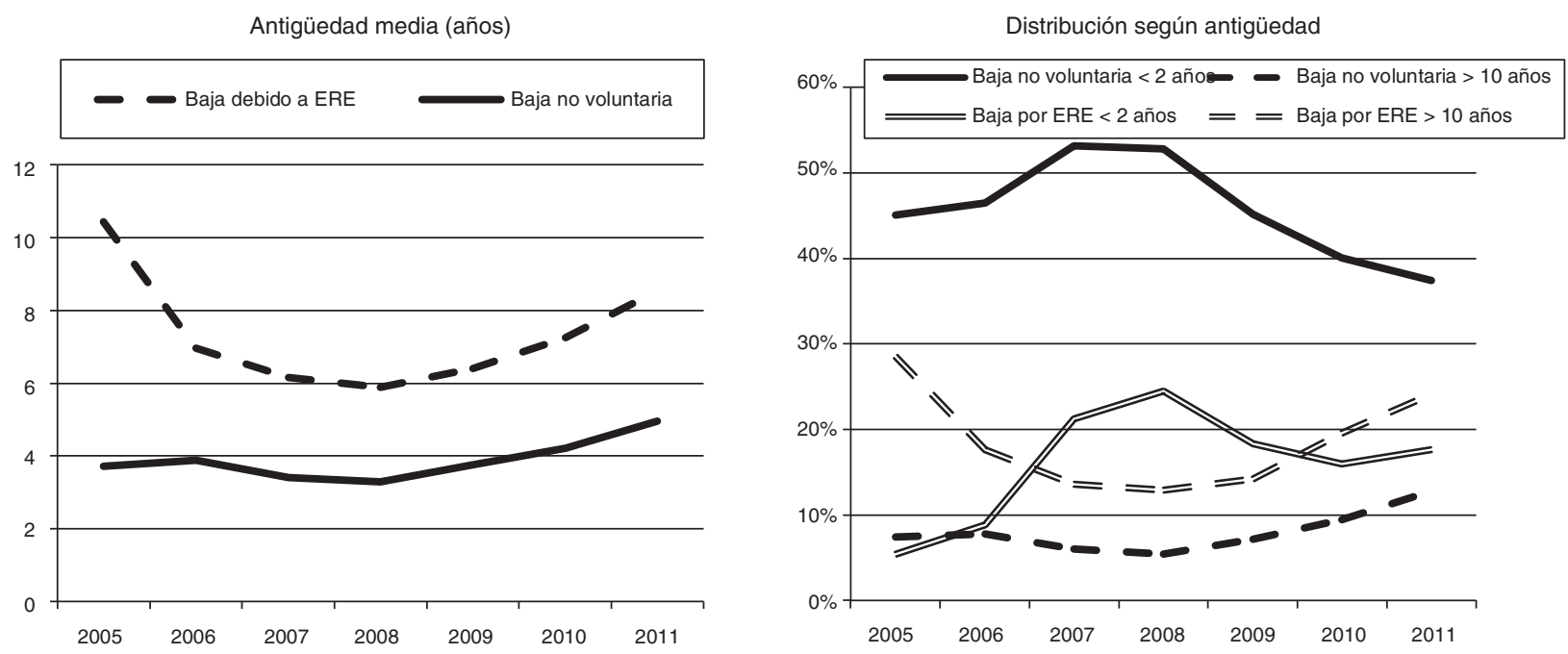

Figura 6 Duración de los contratos terminados.

Fuente: Muestra Continua de Vidas Laborales (ediciones 2005 a 2011). 
cuya duración supera los 10 años, pasando de poco más del $5 \%$ a más del $12 \%$.

Estos datos parecen indicar que, al comienzo de la crisis, en los años 2007 y 2008, las empresas parecen optar por prescindir de los trabajadores con menor antigüedad, siguiendo la regla LIFO («last in, first out»). Hay que tener en cuenta que los datos se refieren únicamente a finalizaciones de contratos indefinidos, puesto que en el análisis no se incluyen los temporales. Por tanto, incluso en el caso de trabajadores indefinidos el coste de despido es asumible si estos trabajadores tienen poca antigüedad. Sin embargo, tras ese primer ajuste, que resulta insuficiente, es necesario despedir empleados que llevan más tiempo trabajando en la misma empresa.

Las indemnizaciones por despido en España son lineales en la antigüedad, pero el incremento es distinto si se trata de un despido procedente o improcedente. Según GarcíaSerrano et al. (2010b), «la regla LIFO es más probable que aparezca si lo normal es la improcedencia que si es la procedencia, pues en el primer caso el incremento marginal de la indemnización con la antigüedad es 45 días de salario (o 33 en el caso de los contratos indefinidos de fomento) mientras que es de 20 días de salario en el segundo caso». Los cambios derivados de la reforma de 2010 pueden alterar la regla LIFO en la medida en que si resulta menos complicado justificar la causa económica del despido, se puede producir un desplazamiento en el peso de las causas del despido de los improcedentes a los procedentes, de forma que la antigüedad en la empresa pierde peso para decidir de qué empleados prescindir.

Como ya mencionamos, la falta de flexibilidad interna y salarial de las empresas para adaptarse a la variante situación económica tiene como resultado el uso del despido como principal medida de ajuste. Dado que en España las indemnizaciones por despido están relacionadas con la antigüedad, el ajuste de los trabajadores sigue una regla LIFO, es decir, los trabajadores despedidos suelen ser los que llevan menos tiempo trabajando en la empresa. El uso mayoritario del despido exprés por el que el empresario incurría en unos mayores costes de despido, a cambio de reducir la incertidumbre, favorecía el uso de la regla LIFO. Cuanto mayores son los costes de despido, mayor es el incentivo del empresario a despedir a los trabajadores con menor antigüedad, independientemente de su productividad. Autores como Malo (2010a) y García-Serrano et al. (2010a, b) señalan que la reforma llevada a cabo en 2010 podría reducir el uso esta regla al clarificar los requisitos del despido objetivo y, por tanto, reducir la incertidumbre del empresario, logrando que la antigüedad tenga un menor peso a la hora de decidir despidos.

Es decir, se podría plantear como hipótesis que la reforma realizada en 2010 reduciría el incentivo a despedir a los trabajadores basándose principalmente en un criterio de antigüedad. Para tratar de comprobar esta hipótesis, se han seleccionado todos los contratos indefinidos en el periodo considerado (tanto los que siguen vigentes como los terminados) y se han agrupado en función de su duración. Para cada uno de los tramos considerados se ha calculado el porcentaje que suponen las bajas no voluntarias.

En la tabla 3 podemos observar que la mayor parte de los contratos indefinidos que se terminan de forma no voluntaria tienen una duración inferior a los 2 años. Por ejemplo, en
2005 , el $15 \%$ de los contratos en dicho tramo causaban baja involuntaria, mientras que cesaban menos del $5 \%$ de los que tenían duración superior a 5 años. Entre 2007 y 2009 dicho porcentaje se eleva hasta casi duplicarse en el caso de los de duración inferior a 2 años, mientras que en los restantes tramos se mantiene relativamente constante. Es decir, los efectos de la crisis en términos de despidos se concentran fundamentalmente en los contratos indefinidos con menor antigüedad. Sin embargo, en los años 2010 y 2011 parece que se produce un cambio, y mientras disminuye la proporción de bajas no voluntarias entre los contratos de menos duración, sube ligeramente en los otros 3 tramos considerados.

Si atendemos a la evolución de la antigüedad media en los contratos finalizados con baja involuntaria y tenemos en cuenta el tipo de contrato (tabla 4), en todo el periodo, la mayor duración del contrato finalizado de forma involuntaria corresponde a los indefinidos ordinarios, con un mínimo de 4 años en 2008 y un máximo de 5,7 en 2011. Entre 2005 y 2008, la antigüedad en los otros 3 tipos de contratos indefinidos se situaba en torno a 2 años. En el caso de las conversiones acogidas al programa de FEE, su duración a partir de 2008 comienza a aumentar rápidamente, alcanzando casi los 5 años en 2011. En las otras 2 modalidades también se produce un incremento de la antigüedad media, si bien no es tan intenso.

Estos datos y los cambios observados en la proporción de bajas no voluntarias según la duración del contrato parecen avalar la hipótesis mencionada previamente, al producirse un desplazamiento en las bajas no voluntarias desde las duraciones más bajas a las que son un poco más largas; por lo tanto, la aplicación de la regla LIFO habría perdido fuerza.

\subsection{Los despidos colectivos}

Como ya se ha visto en la sección anterior, las bajas por ERE son minoritarias, constituyen un pequeño porcentaje del total, aunque se han incrementado sustancialmente desde 2008. El perfil del trabajador afectado por un ERE frente al afectado por una baja no voluntaria difiere de forma importante, al menos en lo que se refiere a la antigüedad. Así, tal y como se puede observar en la figura 6, la duración del contrato es mucho mayor. Del mínimo de 6 años en 2007 y 2008 se pasa a más de 8 años en 2011, lo que supone 3 años más que en el caso de las bajas no voluntarias. De la misma forma, el porcentaje de contratos de duración inferior a 2 años afectados por ERE es sustancialmente menor que el de las bajas no voluntarias, mientras que ocurre lo contrario con los de duración superior a 10 años.

La clarificación de las causas económicas en el caso del despido objetivo llevada a cabo mediante la reforma de 2010 afecta tanto al despido individual como al colectivo. Sin embargo, la necesaria autorización administrativa previa en el caso de los despidos colectivos hace que se difumine el efecto de esta reforma en la regla LIFO, dado que en las negociaciones previas a dicha autorización en la mayoría de los casos se alcanzaban indemnizaciones superiores a los 20 días de salario (Malo, 2012a). Además, otro de los objetivos del periodo de consulta es establecer los criterios a tener en cuenta para la designación de los trabajadores despedidos, lo que influye en diversas características de los despedidos, entre ellas la antigüedad. Por lo tanto, 
Tabla 3 Bajas no voluntarias según duración del contrato (\% sobre número de contratos en cada grupo)

\begin{tabular}{llllll}
\hline & Hasta 2 años & 2 a 5 años & 5 a 10 años & Más de 10 años & Total \\
\hline 2005 & 15,1 & 6,9 & 4,7 & 3,9 & 7,9 \\
2006 & 15,5 & 7,7 & 4,2 & 4,4 & 8,2 \\
2007 & 18,0 & 6,7 & 5,1 & 3,6 & 8,7 \\
2008 & 26,2 & 8,8 & 6,3 & 4,4 & 11,3 \\
2009 & 29,1 & 10,7 & 6,9 & 4,7 & 11,9 \\
2010 & 24,0 & 10,6 & 6,1 & 5,6 & 10,4 \\
2011 & 23,0 & 11,4 & 7,9 & & 11,1 \\
\hline
\end{tabular}

Fuente: Muestra Continua de Vidas Laborales (ediciones 2005 a 2011).

Tabla 4 Antigüedad media de los contratos finalizados con baja involuntaria según tipo de contrato (años)

\begin{tabular}{llllll}
\hline & Indefinido ordinario & Indefinido conversión FEE & Indefinido inicial FEE & Indefinido conversión & Total \\
\hline 2005 & 4,4 & 2,0 & 2,0 & 1,9 & 3,7 \\
2006 & 4,7 & 1,8 & 1,9 & 2,1 & 3,9 \\
2007 & 4,3 & 2,0 & 1,4 & 1,8 & 3,4 \\
2008 & 4,0 & 2,7 & 1,9 & 1,9 & 3,3 \\
2009 & 4,5 & 3,6 & 2,4 & 2,3 & 3,8 \\
2010 & 4,9 & 4,2 & 2,6 & 2,7 & 4,2 \\
2011 & 5,7 & 4,5 & 3,9 & 3,0 & 5,0 \\
\hline
\end{tabular}

Fuente: Muestra Continua de Vidas Laborales (ediciones 2005 a 2011).

la eliminación de la necesidad de autorización administrativa y la supresión de la necesidad de acuerdo con los representantes de los trabajadores ${ }^{11}$ a partir de la entrada en vigor del Real Decreto-Ley 3/2012 probablemente lleve aparejado un cambio de tendencia en la aplicación de la regla LIFO, al aproximar la indemnización real del despido colectivo a la del despido individual y al aumentar la capacidad del empresario para seleccionar qué trabajadores despedir.

\section{Discusión}

El debate sobre los efectos de los costes de despido sobre el empleo ha cobrado relevancia a raíz de la crisis económica que sufrimos, de forma que es uno de los aspectos sobre los que han incidido las reformas habidas en 2010 y 2012. Sin embargo, no es esta la primera vez que una reforma laboral afecta a los costes de despido en los contratos indefinidos. La realizada en 1997 introdujo, para determinados colectivos, el nuevo contrato indefinido de fomento del empleo con una indemnización de 33 días en lugar de 45 en

\footnotetext{
11 Hasta la entrada en vigor de esta norma tampoco era obligatoria la existencia de acuerdo con los representantes de los trabajadores; sin embargo, si se alcanzaba dicho acuerdo la Autoridad Laboral lo autorizaba directamente, y en caso de no alcanzarlo, debía decidir si lo autorizaba o no. Esta regulación suponía un incentivo a las 2 partes, aunque principalmente para el empresario, para alcanzar un acuerdo para asegurarse de que el ERE salía adelante.
}

caso de improcedencia de un despido económico objetivo ${ }^{12}$. Además, las bonificaciones a la contratación indefinida existentes, con una duración de 2 años en la mayor parte de los casos, conducían a que la contratación indefinida resultase más barata que la temporal pero, una vez trascurrido ese tiempo, dejara de compensar la contratación, a menos que a la empresa le interesase mantener al trabajador (Cueto, 2006; Malo y Toharia, 1997). Es decir, los incentivos para utilizar la regla LIFO se derivan tanto de los costes de despido como de las bonificaciones a la contratación indefinida.

Las reformas de 2010 y 2012 afectan a ambos aspectos. Por una parte, cambian la definición de las causas de despido y la indemnización y, por otra parte, los incentivos a la contratación se reducen sustancialmente en términos de los colectivos de trabajadores afectados. La simultaneidad de los cambios, junto con el efecto de la crisis económica y el hecho de que todos los trabajadores se vean afectados, dificulta la realización de una evaluación de los efectos de las reformas, de un análisis causal, que permita identificar cuáles son los factores que conducen a la aplicación o no de la regla LIFO.

A tenor de los datos, tras la reforma de 2010 se observa una relajación de la regla LIFO, con una menor incidencia de los despidos entre los trabajadores de menor antigüedad. Desde el punto de vista empresarial resulta positiva la clarificación del despido por causas económicas, reduciendo la incertidumbre que podía llevar al empresario a

\footnotetext{
12 Se planteó inicialmente por 4 años, pero fue prorrogado posteriormente.
} 
optar por el «despido exprés» (más caro), favoreciendo que sean los trabajadores más productivos y no los más antiguos los que permanezcan en la empresa. Por otra parte, desde el punto de vista social, los trabajadores con una mayor antigüedad tienen cubierto un mayor periodo de cotización de cara a percibir una prestación por desempleo contributiva, lo que implica que tienen un mayor periodo de transición entre empleos cubierto que un trabajador con una menor antigüedad y, presumiblemente, una menor cotización. Sin embargo, los trabajadores de más edad pueden tener más problemas para reincorporarse al mercado de trabajo y mayores probabilidades de convertirse en parados de larga duración.

El análisis realizado aquí pone de manifiesto que se ha producido un cambio en la antigüedad media y, sobre todo, que se ha modificado el porcentaje que suponen los contratos indefinidos menos antiguos sobre el total de despidos. Estos cambios coinciden tanto con la reforma de 2010 como con una intensificación de la crisis económica, de forma que no es posible vincularlos de manera causal a la reforma laboral.

Por otra parte, la reforma de 2012, en la medida en que profundiza en los cambios realizados en 2010 (Malo, 2012b), podría intensificar esta tendencia hacia el despido de trabajadores de mayor antigüedad. En los próximos meses será necesario analizar los datos disponibles para estudiar la evolución de las variables aquí presentadas.

En definitiva, las diferencias en la probabilidad de despido en los contratos indefinidos de menor y mayor antigüedad parecen haberse reducido tras la reforma de 2010 y pueden intensificarse tras la reforma de 2012. Sin necesidad de un contrato único, las reformas han disminuido el uso de la regla LIFO en los despidos, favoreciendo la permanencia de los trabajadores más productivos en la empresa. Debemos tener en cuenta que dicho contrato único solo podría tener efectos en la regla LIFO entre trabajadores temporales e indefinidos, mientras que entre los indefinidos se mantendría, puesto que el incremento no lineal de las indemnizaciones con la antigüedad hace que tenga un mayor efecto la regla LIFO al estar más vinculadas la antigüedad y la estabilidad. Desaparecería la actual dualidad del mercado de trabajo entre trabajadores temporales e indefinidos, pero aparecería una nueva dualidad entre trabajadores con más y menos antigüedad.

\section{Conclusiones}

Tradicionalmente, el despido en España ha seguido la regla LIFO, de manera que son los trabajadores que se han incorporado en último lugar a la empresa los primeros en ser despedidos. Dado que las indemnizaciones por despido en nuestro país están directamente relacionadas con la antigüedad, esta se convierte en la principal pieza a la hora de seleccionar qué trabajadores despedir. El objetivo con el que se ha planteado este trabajo es analizar qué ha ocurrido con las características de los trabajadores despedidos en los últimos años de crisis económica y tras las reformas laborales realizadas.

A partir de datos de la MCVL, entre los años 2005 y 2011 se observa que aunque en todos los años analizados la principal causa de baja de los contratos indefinidos es la baja no voluntaria, a partir de 2009 el porcentaje que suponen estas bajas aumenta hasta superar el $60 \%$ del total. En cuanto a la antigüedad en la empresa de los trabajadores despedidos, observamos que hasta 2008 la antigüedad media era decreciente, alcanzando valores de 3,3 años en dicha anualidad. Sin embargo, en los últimos 3 años ha cambiado la tendencia, alcanzando una antigüedad media de 5 años en 2011. Es decir, parece que en los primeros años de la crisis las empresas seguían la regla LIFO pero que, en años sucesivos, hay un menor uso de esta regla.

Los datos no permiten realizar un análisis causal, por lo que no es posible identificar en qué medida la crisis, los cambios en las bonificaciones o las modificaciones en las indemnizaciones por despido son responsables de los cambios mostrados en el perfil del trabajador despedido. No obstante, la información mostrada sí permite concluir que se ha producido una modificación en el uso de la regla LIFO en el mercado de trabajo de nuestro país.

\section{Bibliografía}

Cebrián, I., Moreno, G., Toharia, L., 2011a. Evaluación microeconómica de los programas de fomento del empleo indefinido a partir de los datos de los servicios públicos de empleo y de la Muestra Continua de Vidas Laborales. Ministerio de Trabajo e Inmigración, Colección Informe y Estudios: Empleo, Madrid.

Cebrián, I., Moreno, G., Toharia, L., 2011b. La estabilidad laboral y los programas de fomento de la contratación indefinida. Revista de Hacienda Pública 198, 103-130.

Cueto, B., 2006. Las ayudas a la contratación en España. Revista de Economía Laboral 3, 87-119.

Desdentado, A., 2010. Crisis y reforma del despido: puntos críticos seguidos de algunas propuestas. Actualidad Laboral 11, $1263-1280$

García Martínez, Malo, M.A., 2007. The strategic use of dismissal legislation: An empirical analysis using Spanish data. European Journal of Law and Economics 23, 151-167.

García-Serrano, C., 2011. Mercado de trabajo y despidos en España. En: Desdentado Bonete, A. (Ed.), Despido y crisis económica. Los despidos económicos tras la reforma laboral: un análisis desde el Derecho y la Economía. Lex Nova, Valladolid.

García-Serrano, C., Malo, M.A., Pérez Infante, J.I., 2010a. Para una reforma laboral negociada. Relaciones Laborales XXVI, 139-163.

García-Serrano, C., Malo, M.A., Pérez-Infante, J.I., 2010b. Dos cuestiones clave de la reforma laboral: despidos y negociación colectiva. Temas Laborales 107, 53-85.

Hospido, L., Jimeno, J.F., febrero 2011. El ajuste de las plantillas de las empresas españolas: el papel de los despidos por causas objetivas. Boletín Económico del Banco de España.

Malo, M.A., 2005. La evolución institucional del despido: una interpretación en términos de accidente histórico. Revista de Historia Económica XXIII, 83-115.

Malo, M.A., 2010a. La reforma laboral de 2010: una discusión de sus componentes básicos. Cuadernos de Mercado de Trabajo 8, 7-13, Disponible en: http://www.sepe.es/contenido/ observatorio/mercado_trabajo/1848-1.pdf

Malo, M.A., 2010b. Costes de despido, temporalidad y reforma laboral en España. Ponencia presentada en el Workshop Temas candentes del mercado de trabajo. Valencia, 15-16 de julio.

Malo, M.A., 2012a. Una primera valoración de la reforma laboral de 2012. Cuadernos de Información Económica 226, 77-82.

Malo, M.A., 2012b. La reforma laboral de 2012: cambios e incertidumbres. Papeles de Economía Española 133, 84-92. 
Malo, M.A., González Sánchez, J.J., 2010. Análisis comparado de los procedimientos de despido: perspectiva económica y jurídica. Ministerio de Trabajo e Inmigración, Madrid.

Malo, M.A., Toharia, L., 1997. Economía y Derecho del Trabajo: las reformas laborales de 1994 y 1997'. Cuadernos Económicos de ICE 63, 155-174.
Malo, M.A., Toharia, L., 2008. La reforma de los despidos de 2002. Revista del Ministerio de Trabajo 76, 111-128.

Toharia, L., Malo, M.A., 2009. ¿Qué se puede esperar de las reformas del mercado de trabajo? En: de Gómez, V. (Ed.), La reforma laboral en España. Editorial Biblioteca Nueva - Fundación Ortega y Gasset, Madrid, cap. 4. 